\title{
Molecularly imprinted solid-phase extraction for the determination of ten macrolide drugs residues in animal muscles by liquid
}

\section{chromatography-tandem mass spectrometry}

Xuqin Song ${ }^{\dagger}$, Tong Zhou ${ }^{\dagger}$ Q Qingying Liu, Meiyu Zhang, Chenying Meng, Jiufeng Li, Limin He (National Reference Laboratory of Veterinary Drug Residues (SCAU), College of Veterinary Medicine, South China Agricultural University, Guangzhou, 510642, China)

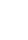

Abstract: A simple and sensitive method based on molecularly imprinted solid-phase extraction coupled with liquid chromatography-tandem mass spectrometry was developed for the determination of the residues of ten macrolide drugs in swine, cattle and chicken muscles samples. The molecularly imprinted polymers (MIPs) were synthesized using tylosin as a template and methacrylic acid as a functional monomer. Samples were extracted with sodium borate buffer solution and ethyl acetate, and purified by the MIP cartridge. The results showed that the cartridge exhibited good recognition performance for macrolides, and better purification effect than the traditional solid-phase extraction cartridges. Recoveries of analytes at three spiking levels 1,5 and $20 \mu \mathrm{g} \mathrm{kg}^{-1}$ ranged from $60.7 \%$ to $100.3 \%$ with the relative standard deviations less than $14 \%$. The limits of detection of the method were between 0.1 and

\footnotetext{
*National Reference Laboratory of Veterinary Drug Residues (SCAU), College of Veterinary Medicine, South China Agricultural University, Guangzhou, Guangdong, PC 510642, China. Tel.: +86 20 85280665; Fax: +86 20 85284896. E-mail address: liminokhe@scau.edu.cn

$\dagger$ The authors contributed equally to this work.
} 
$0.4 \mu \mathrm{g} \mathrm{kg}^{-1}$. The method is useful for the routine monitoring of the residues of 21 macrolide drugs in animal muscles.

22

23 Keywords: Macrolide drugs; Molecularly imprinted polymers; Solid-phase extraction;

24 LC-MS/MS; Animal muscles.

25 


\section{Introduction}

Macrolides are a group of drugs with a large lactone ring that mostly composed of 14 to 16 carbon atoms. Since the discovery of erythromycin (ERY, Fig. S1 for structure) in the 1850s, a large number of macrolide drugs have been developed as the improvement of pharmacological and microbiological effects of macrolides. Nowadays, macrolides have become one of the four most often used anti-infective drugs in the world. They have favorable antibacterial effect on gram-positive, some gram-negative bacteria and mycoplasma. Furthermore, due to the advantages of quick absorption, high oral bioavailability and long half-life, macrolides are widely applied in the clinical treatment of bacterial infections and used as feed additives to prevent diseases (Periti, Mazzei, Mini, \& Novelli, 1989; Zhanel et al., 2001). However, sustained medication and illegal medical additive may lead to drug residues in food animals. The accumulation of drugs in edible animal tissues could be a potential threat to human health through the food chain, which will cause the occurrence of side effects such as gastrointestinal reaction, local stimulation, allergic reaction and hepatotoxicity (Periti, Mazzei, Mini, \& Novelli, 1993). Therefore, supervising and controlling the residues of macrolide drugs in edible animal tissues have been paid a high attention in many countries. In order to ensure human health, the majority of countries have established regulations and set maximum residue limits (MRLs) for macrolide drugs. Furthermore, the macrolide antibiotics have been prohibited from using as feed additives in European Community (EC). With the rapid improvement of 
analytical instruments, it is available to analyze trace drugs and various analytical methods have been gradually developed to detect the drug residues in complex matrices (Kinsella et al., 2009; Reig \& Toldrá, 2008).

The analytical methods of residues of macrolide drugs are mainly targeted at edible animal tissues such as muscle, liver, kidney and fat. Besides, milk, egg and honey are also the interesting animal products for analysts. There are various methods that have been reported for the determination of macrolides in animal muscle samples, including enzyme-linked immunosorbent assay (ELISA) (Galvidis, Lapa, \& Burkin, 2015), capillary electrophoresis (CE) (Zhou, Chen, \& Cassidy, 2000), thin layer chromatography (TLC) (Ahmed, Sree, Abdel-Fattah, Hassan, \& El-Dein Saad, 2013), high performance liquid chromatography (HPLC) (Lee, Yoo, \& Shin, 2013), liquid chromatography-tandem mass spectrometry (LC-MS/MS) (Dickson, 2014). Among these analytical methods, HPLC and LC-MS/MS are the two most widely used and efficient methods due to the availability and high accuracy in different complex matrices. Impurities such as protein and fat existed in animal tissues samples will not only pollute the analytical instruments, which leads to the decrease of the sensitivity, but also affect the separation of target analytes. With the purpose of decreasing the interference of impurities, specific pretreatment measures such as pressurized liquid extraction (PLE) (Tao et al., 2012), solid phase extraction (SPE) (Freitas, Barbosa, \& Ramos, 2015), matrix solid-phase dispersion (MSPD) (García-Mayor, Gallego-Picó, Garcinuño, Fernández-Hernando, \& Durand-Alegría, 2012), solid phase micro-extraction (SPME) (McClure \& Wong, 2007), QuEChERS (QUick, Easy, 
CHeap, Effective, Rugged and Safe) (Kang et al., 2014), have been taken to achieve better separation, purification and preconcentration of target drugs. However, the trace amounts of drug residues, complex matrices and the interference of impurities are still the challenges in analysis. To overcome such difficulties, novel pretreatment techniques such as immunoaffinity chromatography (Zhao, Lin, Song, Pan, \& Wang, 2011) and molecular imprinting (Siemann, Andersson, \& Mosbach, 1997) have been introduced in the selective retention of drugs and the powerful elimination of impurities.

Molecular imprinting technology (MIT) is an emerging and increasingly applied technique that originally derived from the immunological mechanism of the receptor and molecular recognition. Molecularly imprinted polymers (MIPs) are synthesized using the template, functional monomer, cross-linker and initiator, which form the corresponding spatial structure and binding site. Once the template is removed, the MIPs will leave behind a cavity, whose spatial structure and binding site are complementary in size and shape with the target template. Due to the "lock-key" relationship, the MIPs possess the characteristic of selectivity towards the target molecules, and in a consequence can specifically recognize the template or analogues. As a novel molecular recognition material, molecularly imprinted polymers are widely used in the fields of environmental monitoring, bioanalysis and food detection, owing to the selective enrichment characteristic of the MIPs which can specifically concentrate trace amounts of target compounds such as pesticides, traditional Chinese medicine, veterinary drugs or environmental toxins from complicated matrix samples 
(Hantash et al., 2006; Cirillo et al., 2011; Zhang et al., 2006). In terms of drugs residues analysis in food, molecularly imprinted polymers are generally used as the materials of solid phase extraction in the sample cleanup step. Considerable attention has focused on MIP solid phase extraction (MISPE) method for purification and preconcentration of macrolides drugs residues in samples. The molecularly imprinted polymers of erythromycin were prepared by non-covalent bulk polymerization and successfully applied to the cleanup and enrichment of the ERY in pig muscle (Song et al., 2008). The MIPs exhibited powerful affinity toward template molecule with the maximum adsorption amount of $72.09 \mathrm{~g} \mathrm{~kg}^{-1}$ and the recoveries of ERY were more than $80 \%$. It is suggested that the MIPs as solid phase extraction sorbents had high selective performance and could easily absorb the ERY in tissue extracts and efficiently remove the impurities co-extracted with ERY. Piletsky et al. ( Piletsky et al., 2004) synthesized the MIPs of tylosin (TYL, Fig. S1 for structure) that could rebind with the template or related metabolites. Furthermore the MIP could adsorb tylosin from complex matrices with easy removal of lipids and other impurities. Zhang et al. (Zhang et al., 2011) reported that the multi-walled carbon nanotube-molecularly imprinted polymer of ERY prepared was used as solid-phase extraction sorbent, and exhibited good selectivity toward template molecule. The recoveries in chicken muscle sample were between $85.3 \%$ and $95.8 \%$. To the best of our knowledge, researches on molecularly imprinted solid-phase extraction mainly focused on the detection of the template or an analogue, while it's feasible for finding a MIP that can purify and enrich a class of structural analogues in accordance with the class-specific 
recognition sites in the polymers.

The purpose of the present study is to synthesize a class-selective MIP using tylosin as a virtual template and then used as the selective absorbent for packing SPE cartridge. Finally, a simple and sensitive method based on MISPE combining with LC-MS/MS was established for the simultaneous determination of the residues of ten macrolides (Azithromycin (AZI), tulathromycin (TUL), tilmicosin (TIL), erythromycin (ERY), kitasamycin (KIT), spiramycin (SPM), roxithromycin (ROX), josamycin (JOS), clarithromycin (CLA), and medecamycin (MED)) in swine, cattle and chicken muscles samples. The proposed method is appropriate to clean up, enrich and determine macrolide drugs residues in complex biological matrix samples.

\section{Experimental}

\subsection{Reagents and materials}

The required template tylosin was obtained from Hengtong Guanghua (Xian, China). The synthetic materials methacryclic acid (MAA) and 2,2'-Azobisisobutyronitrile (AIBN) were from Kermel Chemical Reagents Development Center (Tianjin, China). Ethylene glycol dimethacrylate (EGDMA) was purchased from Sigma-Aldrich (St. Louis, MO, USA). MAA and EGDMA were removed inhibitor by active carbon before they were used.

The purity of each reference standard (Fig. S1 for structure) was more than $90 \%$. Azithromycin, tulathromycin, tilmicosin, erythromycin, kitasamycin and tiamulin (TIA) were from Sigma Chemicals Co. (St. Louis, MO, USA). Spiramycin, 
roxithromycin, josamycin and clarithromycin were obtained from European Pharmacopoeia (EDQM, Strasbourg, France) and medecamycin was purchased from China Institute of Veterinary Drug Control (Beijing, China). Each of the appropriate standards was dissolved in methanol to prepare the stock solutions at the concentration of $1000 \mathrm{mg} \mathrm{L}^{-1}$. The stock solutions were stored in volumetric flask and could keep stable for 6 months at $4{ }^{\circ} \mathrm{C}$. The working solutions of ten macrolides (100 $\mathrm{mg} \mathrm{L}^{-1}$ ) were prepared monthly by diluting the stock solutions with acetonitrile.

Acetonitrile, methanol and formic acid were chromatographic grade and purchased from Fisher Scientific (Fairlawn, New Jersey, USA). Other reagents including ethyl acetate, acetone and ammonia, etc. were analytical grade or better and purchased from Guangzhou Chemical Reagent Factory (Guangzhou, China). Oasis HLB (60 mg, 3 mL) SPE cartridge was obtained from Waters Co. (Milford, MA, USA), $\mathrm{C}_{18}$ (200 mg, $3 \mathrm{~mL}$ ) and SCX (60 mg, $3 \mathrm{~mL}$ ) SPE cartridges were purchased from Agilent Technologies Co. (Santa Clara, CA, USA). De-ionized water was obtained by Milli-Q water system (Molsheim, France).

\subsection{Preparation of the imprinted polymers}

The MIPs were synthesized according to our method previously reported (Zheng et al., 2011). Briefly, $1.0 \mathrm{mmol}$ tylosin was dissolved in $6 \mathrm{~mL}$ chloroform and then sonicated for 5 min. 8 mmol MAA was added into the dispersion and incubated at $4{ }^{\circ} \mathrm{C}$ for 4 h. 20 mmol cross-linker EGDMA and $20 \mathrm{mg}$ initiator AIBN were added. After sonication for $5 \mathrm{~min}$, the mixture was purged with nitrogen for 5 min to remove oxygen. The polymerization reaction would accomplish by incubating the mixture at 
158

159

160

161

162

163

164

165

166

167

168

169

170

171

172

173

174

175

$60{ }^{\circ} \mathrm{C}$ for $24 \mathrm{~h}$ in a water bath. After grinding and sieving, the polymers were sedimented with acetone to discard the tiny particles. The template was removed by methanol-acetic acid (90:10, v/v) and finally the polymers were dried under vacuum at $60^{\circ} \mathrm{C}$.

\subsection{Filling amount of MISPE cartridge}

The polymers particles were packed into $1 \mathrm{~mL}$ empty SPE cartridge at the amount of 10, 20, 30 and $40 \mathrm{mg}$, respectively. Recovery test was performed with blank muscles matrices at the spiked concentration of $5 \mu \mathrm{g} \mathrm{kg}^{-1}$ of ten macrolides and the procedure of SPE was conducted in the optimum conditions.

\subsection{MISPE conditions}

The cartridges were firstly conditioned by $1 \mathrm{~mL}$ methanol. For the optimal loading solvents, $2 \mathrm{~mL}$ of methanol, ethyl acetate and different proportion of acetonitrile in water $(5 \%, 10 \%, 20 \%$ and $30 \%, v / v)$ were successively evaluated. In the process of washing, methanol, and different proportion of methanol in water (5\%, $10 \%$ and $30 \%, v / v$ ) were applied to remove impurities. The analytes were eluted with methanol and different ratios of ammonia in methanol ( $3 \%, 5 \%$ and $8 \%, v / v)$.

\subsection{Sample preparation}

Swine, cattle and chicken muscles samples were purchased from local supermarkets (Guangzhou, China). After homogenization, all samples were detected no macrolides residuals and kept at $-20{ }^{\circ} \mathrm{C}$. Recovery test was carried out at three spiked concentration levels of 1,5 and $20 \mu \mathrm{gg}^{-1}$.

\subsubsection{Extraction}



centrifuge tube (for recovery test, the samples were spiked by the working solution of ten macrolides and incubated for $30 \mathrm{~min}$ at room temperature to make sure the permeation of the drugs into the matrix). $5 \mathrm{~mL}$ sodium borate buffer solution $(\mathrm{pH}=10)$ and $5 \mathrm{~mL}$ ethyl acetate were used for the extraction of the analytes. After shaken for $30 \mathrm{~min}$, the samples were centrifuged at $9000 \times \mathrm{g}$ for $5 \mathrm{~min}$ at $4{ }^{\circ} \mathrm{C}$ and the supernatants were transferred into a new centrifuge tube. Then add an additional 5 $\mathrm{mL}$ ethyl acetate into the residues and repeat the extraction procedure above. All the supernatants were combined and evaporated to near dryness. The residues were dissolved in $2 \mathrm{~mL}$ of $20 \%$ acetonitrile in water and then the solution was defatted with hexane for cleanup.

\subsubsection{Clean up}

The MISPE cartridge was first conditioned according to the optimum procedures described above, and the loading solution was passed through the MISPE cartridge at interferences and then the cartridge was dried via using a low positive pressure. $1 \mathrm{~mL}$ of $5 \%$ ammonia in methanol was used to elute analytes. The eluates were evaporated to near dryness at $40{ }^{\circ} \mathrm{C}$ under a gentle stream of nitrogen. The residues were re-dissolved in $1 \mathrm{~mL}$ of $20 \%$ methanol in water (containing $0.1 \%$ formic acid) 
USA) coupled with an Applied Biosystem API 4000 triple quadrupole mass

spectrometer (Foster City, CA, USA). Chromatographic separation was performed

with the following linear gradient elution program: 0.0 - 5.0 min $10 \%-60 \%$ A; $5.0-$ $7.0 \min 60 \%-45 \%$ A; $7.0-7.01 \min 45 \%$ - 10\% A; 7.01 - 15 min 10\% A. The total runtime was $15 \mathrm{~min}$ and the column temperature was maintained at $35{ }^{\circ} \mathrm{C}$. The injection volume was $10 \mu \mathrm{L}$ and the flow rate was maintained at $0.25 \mathrm{~mL} \mathrm{~min}^{-1}$. parameters were optimized. $1 \mathrm{mg} \mathrm{L}^{-1}$ individual standard solutions were introduced directly into the mass spectrometer at $0.01 \mathrm{~mL} \mathrm{~min}^{-1}$ by a syringe pump (Harvard Apparatus, Holliston, MA). As macrolide antimicrobials are weak basic compounds, the mass analysis was carried out under the positive electrospray ionization mode. The optimum conditions of multiple reaction monitoring (MRM) were carried out at the following parameters: ion spray voltage (IS), $5500 \mathrm{~V}$; auxiliary gas (GS1 and GS2), 65 and 60 psi, respectively; curtain gas (CUR), 30 psi; ion source temperature 219 (TEM), $600{ }^{\circ} \mathrm{C}$. The optimal mass spectrometric parameters for each compound, 220 including MRM transitions, declustering potential (DP) and collision energy (CE), are 221 given in Table 1. The dwell time of each transition was set to $50 \mathrm{~ms}$. Control of the system, data acquisition and data analysis were all carried out using the Analyst 1.5.2 software (Applied Biosystems). 


\section{Results and discussion}

226

227

228

\subsection{Preparation of the MIP and MISPE cartridge}

\subsubsection{Preparation of the MIP}

The formation of MIPs is a very complex process, which varies with the different kinds of template, monomer, cross-linking and other necessary materials. Generally molecular imprinting methods are broadly divided into two types. One is covalent molecular imprinting, in which the template connects with functional monomer through covalent bonds. The other is via intermolecular interactions such as hydrogen bond, electrostatic interactions or other non-covalent bonds to form non-covalently molecularly imprinted polymers. In this study, the MIPs were synthesized with tylosin as the template and MAA as the functional monomer by non-covalent polymerization. Tylosin is affiliated to 16-member ring macrolide antibiotics, and is composed of two glycosyl groups and a large lactone. The carboxyl group in the MAA (functional monomer) interacted with the hydroxyl and carbonyl group of tylosin (template) through hydrogen bond, thus a specific binding site would be formed in the polymers. After tylosin (template) was removed, the corresponding cavity, which was complementary to template or (and) its analogues, was left in the polymers.

\subsubsection{Optimization of the MISPE cartridge}

Due to the certain number of cavities, the filling amount had a direct impact on the adsorption capacity of the MISPE cartridge for target drugs. For obtaining high recoveries and appropriate permeability, the optimization of the filling amount of 
MISPE cartridge is necessary. The results of the relevant experiments showed that the recoveries of target analytes increased with increasing the filling amount of the MIP in the range from $10 \mathrm{mg}$ to $40 \mathrm{mg}$. The highest recovery was obtained when the filling amount of the MIP was up to $20 \mathrm{mg}$. When the filling amount of the MIP was over 20 mg, the permeability of the MIP cartridge was rapidly decreased and recoveries of major macrolides drugs except KIT and MED (keeping about 80\%) decreased weakly. Especially, the recoveries of ERY and SPM were below 50\%. Considering the recoveries of analytes and appropriate flow rate of the cartridge, $20 \mathrm{mg}$ of the MIP was filled into the cartridge for the following experiment.

\subsubsection{Optimization of the MISPE conditions}

Owing to the important role in avoiding interference of impurities and enriching target compounds, the procedures of SPE (loading, washing and eluting) should be optimized to acquire more effective separation and concentration. Figure 1 reveals the effect of different loading solvents on recoveries. When ethyl acetate or methanol was used as the loading solvent, the recoveries of ERY, CLA, MED and JOS were all below $50 \%$. When the system of acetonitrile and water was used as the loading solution, the recoveries of ten analytes except for JOS gradually increased with increasing the percentage of acetonitrile. While too large acetonitrile in loading solution led to a significant decrease of recoveries for most macrolide drugs. Except for TUL, TIL and JOS, the recoveries of ten macrolide drugs in $20 \%$ acetonitrile aqueous solution were more than those in the other three different percentages of acetonitrile in water. As a consequence, $20 \%$ acetonitrile in water was selected as the 
loading solution.

In order to increase the specific interactions between binding sites in the MIPs and target analytes, and simultaneously decrease the cross-reactivity of complex matrix, the washing step was very significant in MISPE procedure (Jiang et al., 2009). The polymers that were synthesized via non-covalent imprinting should remove impurities in mild conditions. The characteristics of the washing solution such as polarity and $\mathrm{pH}$ will affect the retention of the drugs. The experimental results showed that when methanol was applied to wash impurities, recoveries of TUL, MED and JOS were below 50\%. The recoveries of target analytes improved obviously with the decrease of methanol in washing solution except that the recoveries for SPM and TIL slightly decreased. Finally, $10 \%$ methanol in water was selected as the washing solution to remove more organic impurities in the extracts.

The elution step is the final and most important procedure in solid phase extraction, because analytes should be sufficiently desorbed from the SPE cartridge. In the elution step of solid-phase extraction, ammonia in methanol was often utilized to elute weak basic analytes. On basis of macrolide drugs are weak organic bases, different percentages of ammonia in methanol were used to elute target compounds. The results showed that the percentage of ammonia in methanol from $5 \%$ to $8 \%$ could elute macrolide drugs effectively, while pure methanol and 3\% ammonia in methanol could not elute TUL completely. In consideration of efficient evaporation of the eluent, $5 \%$ ammonia in methanol was more suitable as the eluting solution, which provided recoveries of ten macrolide drugs between $78.2 \%$ and $98.4 \%$. 
290

291

292

293

294

295

296

297

298

299

300

301

302

303

304

305

306

307

308

309

310

311

\subsection{Sample preparation}

\subsubsection{Extraction}

On basis of the characteristics of alkalescence and lipophilic molecules, various solvents were applied to the extraction of macrolide compounds. The common solvents such as methanol (Wu et al., 2013), acetonitrile (Błądek, Posyniak, Gajda, Gbylik, \& Żmudzki, 2012), Tris buffer (Dubois, Fluchard, Sior, \& Delahaut, 2001), phosphate buffer solution (García-Mayor, Garcinuño, Fernández-Hernando, \& Durand-Alegría, 2006) or ethyl acetate (Wang et al., 2014) were reported. In this study, the extraction efficiency of three extraction solvents including methanol, acetonitrile and ethyl acetate was compared. Based on the principle of similarity and intermiscibility, as far as the alkalescent macrolide drugs are concerned, their residues in animal muscles samples should be easily extracted by ethyl acetate in alkalescent media. Thus, before extraction using ethyl acetate, the composites of muscles were dispersed in sodium borate buffer solution ( $\mathrm{pH}=10.0)$. As shown in Fig. 2, in brief, extraction efficiency of ethyl acetate is the highest, the efficiency of acetonitrile is also satisfactory, and that of methanol is the lowest. Ethyl acetate as extraction solvent, the recoveries of ten macrolide analytes ranged from $72.5 \%$ to $93.4 \%$. Acetonitrile as extraction solvent, the recoveries of ten macrolide analytes ranged from $68.0 \%$ to 85.0\%. However, the recoveries of ERY, CLA and MED were below 60\% when methanol was used as the extraction solvent. Moreover, the extracts of methanol contained more impurities, which led to the difficulty of solid phase extraction. Therefore, the system of sodium borate buffer solution $(\mathrm{pH}=10.0)$ and ethyl acetate 
was selected for the extraction of the residues of macrolides from muscles samples.

\subsubsection{Clean up}

Muscles samples contain various endogenous impurities, such as lipids and protein, which will decrease recoveries and affect separation and analysis. Utilizing appropriate SPE cartridges will remove interferences from complex matrices and concentrate the target analytes. Several types of SPE cartridges are available for cleanup and concentration of macrolides, such as Oasis HLB (Horie, Takegami, Toya, \& Nakazawa, 2003), $\mathrm{C}_{18}$ (Dickson, 2014), MCX (Boner, Gottschall, \& Kim-Kang, 2011) and SCX (Horie et al., 1998). Three different types of SPE cartridges $\left(\mathrm{C}_{18}\right.$, Oasis HLB and SCX) were compared with the MISPE cartridge in this experiment. As for $\mathrm{C}_{18}$ cartridge, $2 \mathrm{~mL}$ of $20 \%$ acetonitrile in water was used as the loading solution, $3 \mathrm{~mL}$ water and $3 \mathrm{~mL} 5 \%$ methanol in water as the washing solvents, $5 \mathrm{~mL}$ of $5 \%$ ammonia in methanol as the elution solvent. As for Oasis HLB cartridge, except that 2 $\mathrm{mL}$ phosphate buffer solution $(0.1 \mathrm{M}, \mathrm{pH}=8.0)$ was applied to dissolve the residues, washing solvent and elution solvent were the same as the $\mathrm{C}_{18}$ cartridge. As for SCX cartridge, $0.3 \%$ metaphosphoric acid-methanol (7:3, v/v) was applied to dissolve the residues and then passed through the cartridge, which was previously conditioned with $3 \mathrm{~mL}$ of methanol and $3 \mathrm{~mL}$ of $0.1 \mathrm{M} \mathrm{KH}_{2} \mathrm{PO}_{4}(\mathrm{pH}=4.4), 3 \mathrm{~mL}$ of water and methanol as the washing solvents, respectively. Finally, $5 \mathrm{~mL}$ of $5 \%$ ammonia in methanol was used to elute target analytes.

This experiment was accomplished in swine muscle at the spiked concentration of $5 \mu \mathrm{gg}^{-1}$. As shown in Fig. 3, the MISPE cartridge revealed good retention 
efficiency for the ten macrolides. The recoveries on the MISPE were obtained from $69.4 \%$ to $92.2 \%$ and were slightly higher than those obtained from the $\mathrm{C}_{18}$ cartridge except for TIL and CLA. When the HLB cartridge was applied to purification, the retention efficiency of AZI, SPM and TIL was poor and their recoveries were only about $60 \%$. As for SCX cartridge, except for TUL, TIL, SPM and AZI, other macrolides were not retained well in the cartridge and the recoveries were approximately $60 \%$. Compared with Oasis HLB, $\mathrm{C}_{18}$ and SCX cartridges, the recovery of the MISPE cartridge for tiamulin whose chemical structure is different from macrolide compounds was below $60 \%$. This phenomena hinted that macrolide drugs could be retained well in the MIP as the result of the existence of memory cavities with the template molecules such as size, shape and binding sites (Dai, Zhou, Zhang, Liu, \& Zhang, 2011). Tiamulin is affiliated to the diterpenoids, which does not match the corresponding binding sites in the MIP and was washed off from MISPE cartridge.

\subsection{Method validation}

In order to validate the proposed method, confirmation, selectivity, linearity, recovery, precision of intra-day and inter-day, limit of detection (LOD) and limit of quantification (LOQ) were evaluated.

In accordance with Commission Decision 2002/657/EC (Commission, 2002), if a new method is to be deemed confirmatory, identification of a target analyte must meet the tolerance for the retention time, identification points (IPs) of the analyte and relative ion ratios of selected MRM transitions. In this study, all ten analytes were 
monitored in MRM mode. We monitored one precursor and two product ions (quantification and confirmation) for each analyte providing 4 IPs. Confirmation was carried out by comparison of the ratio of the relative intensity of confirmation transition and quantification transition of target analyte in pure solvent and matrix

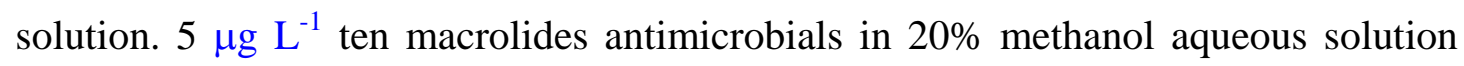
(containing $0.1 \%$ formic acid) and the extracts of swine muscle (as a matrix-matched standard solution) were tested. The results showed that the relative deviations of ion ratios of all ten analytes meet the specific requirements stated in EC validation criteria. The mean ion ratios of all target analytes in pure solvent (20\% methanol aqueous solution (containing $0.1 \%$ formic acid)) and the reconstitution solution of extracts of swine muscle were summarized in Table 1.

Selectivity of the method was performed by analyzing more than 60 blank samples collected different swine, cattle and chicken muscles and observing the existence of the matrix interferences at the elution zone of each analyte. There were no interferences' peaks within the $2.5 \%$ margin of the retention time of the target compounds, indicating good selectivity of this developed method. The typical MRM chromatograms of extracts of blank swine muscle were shown in Fig. 4. The MRM chromatograms of extracts of cattle and chicken blank muscles were presented in supplementary data (Fig. S2).

The calibration curves were prepared by adding pure standards to blank swine, cattle and chicken muscles in order to obtain the spiked concentrations of $0.1,0.5,1.0$, 5.0, 10, 20, 100 and $200 \mu \mathrm{g} \mathrm{kg}{ }^{-1}$. Five replicates were used at each spiking 
concentration. The linear equations were originated from using the peak area of analyte versus the corresponding concentration in the matrix solution. As shown in Table 2, the results revealed there were good linearity of calibration curves for all ten compounds and the correlation coefficients $\left(r^{2}\right)$ were higher than 0.99 . Intra-day and inter-day recoveries were usually used to describe the accuracy of the method. Three kinds of animal muscles described above were performed at three spiked concentration levels ( 1,5 and $20 \mu \mathrm{gg}^{-1}$ ). Each spiking levels were prepared in six replicates and detected in consecutive three days. Ten macrolides were analyzed by LC-MS/MS after extraction and further cleanup with the MISPE cartridge.

Recoveries for all analytes were calculated by the corresponding linear equations. As shown in Table 2, the recoveries of TUL and SPM were relatively lower (below 70\%) while those of MED, JOS and KIT were higher (more than 75\%). Mean recoveries of ten target analytes were in the range of $60.7 \%$ to $100.3 \%$. The typical MRM chromatograms with electronic ionspray source in positive mode achieved from the extracts of blank swine matrix at the spiked concentration of $5 \mu \mathrm{kg}^{-1}$ were illustrated (Fig. 4).

Precisions (intra-day, inter-day) were carried out in terms of analyzing each kind of muscle in six replicates and determined in three consecutive days. The precision was expressed as relative standard deviation (RSD). As for three kinds of animal muscles, intra-day RSDs were ranged from $0.2 \%$ to $14 \%$ and inter-day RSDs were from $2.4 \%$ to $14 \%$. The results conformed to the requirement of residual analysis in edible animal tissues samples. 
401

402

generally expressed as LOD and LOQ. The signal to noise ratio $(S / N)$ is utilized to determine LOD and LOQ. In this study, the LOD and LOQ were estimated by the spiking at a series of concentrations of the target analytes in muscles matrices.

Generally, the LOD is the corresponding concentration when $S / N$ is $\geq 3$ and the LOQ is estimated in terms of the criterion of $S / N \geq 10$. The data are given in Table 2 . The LODs of ten analytes ranged from $0.1 \mu \mathrm{g} \mathrm{kg}$-1 to $0.4 \mu \mathrm{g} \mathrm{kg}{ }^{-1}$, and the LOQs were in the range of $0.3-1.0 \mu \mathrm{gg}^{-1}$. The MRM chromatograms with electronic ionspray source in positive mode achieved from the extracts of blank swine, cattle and chicken muscles matrices at the spiked concentration of the LOQ of each macrolide drugs were presented in supplementary data (Fig. S3 and Fig. S4). The results indicated the sensitivity of the proposed method for the determination of ten macrolide drugs residues in animal muscle samples was acceptable.

\section{Conclusions}

A molecularly imprinted polymer was synthesized using tylosin as the virtual template, and the capacity of rebinding the analogues of the template was ideal.

\section{Compared with the conventional SPE cartridges, the MISPE cartridge prepared was} higher selective and obtained higher recoveries for ten macrolides drugs. The method developed can be suitable for sensitive, reproducible and simultaneous determination of the residues of ten macrolide drugs in animal muscle tissues samples.

\section{Acknowledgements}

The authors are grateful to the financial support from the National Science 
422 Foundation of China (31372476), the Program for Changjiang Scholars and

423 Innovative Research Team in University (IRT13063) and Science and Technology

424 Program Project of Guangzhou (2014J4100190).

425

426

427

428

430

431

432

433

434

435

436

437

438

439

440

441
References

Ahmed, M., Sree, Y., Abdel-Fattah, S., Hassan, N., \& El-Dein Saad, M. (2013). Determination of tylosin, spiramycin, and erythromycin residues in Egyptian buffaloes' meat by thin-layer chromatography-bioautography. JPC-Journal of Planar Chromatography-Modern TLC, 26(5), 409-416.

Błądek, T., Posyniak, A., Gajda, A., Gbylik, M., \& Żmudzki, J. (2012). Multi-class procedure for analysis of antibacterial compounds in eggs by liquid chromatography-tandem mass spectrometry. Bulletin of the Veterinary Institute in Pulawy, 56(3), 321-327.

Boner, P. L., Gottschall, D. W., \& Kim-Kang H. (2011). Determination and confirmation of tulathromycin residues in bovine liver and porcine kidney via their common hydrolytic fragment using high-performance liquid chromatography/tandem mass spectrometry. Journal of AOAC International, 94(2), 436-445(410).

Cirillo, G., Curcio, M., Parisi, O. I., Puoci, F., lemma, F., Spizzirri, U. G., Restuccia, D., \& Picci, N. (2011). Molecularly imprinted polymers for the selective extraction of glycyrrhizic acid from liquorice roots. Food chemistry, 125(3), 1058-1063.

Commission, E. U. (2002). European Commission Decision 2002/657/EC. In E. U. Commission (Ed.), (pp. 8-36): Official Journal of the European Communities.

Dai, C. M., Zhou, X. F., Zhang, Y. L., Liu, S. G., \& Zhang, J. (2011). Synthesis by precipitation 

polymerization of molecularly imprinted polymer for the selective extraction of diclofenac from water samples. Journal of Hazardous Materials, 198(2), 175-181.

Dickson, L. C. (2014). Performance characterization of a quantitative liquid chromatography-tandem mass spectrometric method for 12 macrolide and lincosamide antibiotics in salmon, shrimp and tilapia. Journal of Chromatography B, 967, 203-210.

Dubois, M., Fluchard, D., Sior, E., \& Delahaut, P. (2001). Identification and quantification of five macrolide antibiotics in several tissues, eggs and milk by liquid chromatography-electrospray tandem mass spectrometry. Journal of Chromatography B, 753(2), 189-202.

Freitas, A., Barbosa, J., \& Ramos, F. (2015). Multidetection of antibiotics in liver tissue by ultra-high-pressure-liquid-chromatography-tandem mass spectrometry. Journal of Chromatography $B, 976,49-54$.

Galvidis, I., Lapa, G., \& Burkin, M. (2015). Group determination of 14-membered macrolide antibiotics and azithromycin using antibodies against common epitopes. Analytical biochemistry, 468, 75-82.

García-Mayor, M., Gallego-Picó, A., Garcinuño, R., Fernández-Hernando, P., \& Durand-Alegría, J. (2012). Matrix solid-phase dispersion method for the determination of macrolide antibiotics in sheep's milk. Food Chemistry, 134(1), 553-558.

García-Mayor, M. A., Garcinuño, R. M., Fernández-Hernando, P., \& Durand-Alegría, J. S. (2006). Liquid chromatography-UV diode-array detection method for multi-residue determination of macrolide antibiotics in sheep's milk. Journal of Chromatography A, 1122(1-2), 76-83. 
Horie, M., Saito, K., Ishii, R., Yoshida, T., Haramaki, Y., \& Nakazawa, H. (1998). Simultaneous determination of five macrolide antibiotics in meat by high-performance liquid chromatography. Journal of Chromatography A, 812(1-2), 295-302.

Horie, M., Takegami, H., Toya, K., \& Nakazawa, H. (2003). Determination of macrolide antibiotics in meat and fish by liquid chromatography-electrospray mass spectrometry. Analytica chimica acta, 492, 187-197.

Jiang, T., Zhao, L., Chu, B., Feng, Q., Yan, W., \& Lin, J.M. (2009). Molecularly imprinted solid-phase extraction for the selective determination of $17 \beta$-estradiol in fishery samples with high performance liquid chromatography. Talanta, 78(2), 442-447.

Kang, J., Fan, C.L., Chang, Q.Y., Bu, M.N., Zhao, Z.Y., Wang, W., \& Pang, G.F. (2014). Simultaneous determination of multi-class veterinary drug residues in different muscle tissues by modified QuEChERS combined with HPLC-MS/MS. Analytical Methods, 6(16), 6285-6293.

Kinsella, B., O’Mahony, J., Malone, E., Moloney, M., Cantwell, H., Furey, A., \& Danaher, M. (2009). Current trends in sample preparation for growth promoter and veterinary drug residue analysis. Journal of Chromatography A, 1216(46), 7977-8015.

Lee, S.H., Yoo, M., \& Shin, D.B. (2013). Determination of four macrolide antibiotics residues in chicken muscle using high-performance liquid chromatography. Journal of Food Hygiene and Safety, 28(1), 19-23.

McClure, E. L., \& Wong, C. S. (2007). Solid phase microextraction of macrolide, trimethoprim, and sulfonamide antibiotics in wastewaters. Journal of Chromatography A, 1169(1), 53-62. 
Periti, P., Mazzei, T., Mini, E., \& Novelli, A. (1989). Clinical pharmacokinetic properties of the macrolide antibiotics. Clinical pharmacokinetics, 16(4), 193-214.

Periti, P., Mazzei, T., Mini, E., \& Novelli, A. (1993). Adverse effects of macrolide antibacterials. Drug safety, 9(5), 346-364.

Piletsky, S., Piletska, E., Karim, K., Foster, G., Legge, C., \& Turner, A. (2004). Custom synthesis of molecular imprinted polymers for biotechnological application: Preparation of a polymer selective for tylosin. Analytica chimica acta, 504(1), 123-130.

Reig, M., \& Toldrá, F. (2008). Veterinary drug residues in meat: Concerns and rapid methods for detection. Meat science, 78(1), 60-67.

Siemann, M., Andersson, L. I., \& Mosbach, K. (1997). Separation and detection of macrolide antibiotics by HPLC using macrolide-imprinted synthetic polymers as stationary phases. The Journal of antibiotics, 50(1), 89-95.

Song, S., Wu, A., Shi, X., Li, R., Lin, Z., \& Zhang, D. (2008). Development and application of molecularly imprinted polymers as solid-phase sorbents for erythromycin extraction. Analytical and bioanalytical chemistry, 390(8), 2141-2150.

Tao, Y., Yu, G., Chen, D., Pan, Y., Liu, Z., Wei, H., Peng, D., Huang, L., Wang, Y., \& Yuan, Z. (2012). Determination of 17 macrolide antibiotics and avermectins residues in meat with accelerated solvent extraction by liquid chromatography-tandem mass spectrometry. Journal of Chromatography $B, 897,64-71$.

Wang, Z., Song, X., Zhou, T., Bian, K., Zhang, F., He, L., \& Liu, Q. (2014). Simultaneous determination of ten macrolides drugs in feeds by high performance liquid chromatography with evaporation light scattering detection. Rsc Advances, 5, 1491-1499. 
510

511

512

513

514

515

516

517

518

519

520

521

522

523

524

525

526

527

528

529

530

531

Wu, J., Qian, Y., Zhang, C., Zheng, T., Chen, L., Lu, Y., \& Wang, H. (2013). Application of graphene-based solid-phase extraction coupled with ultra high-performance liquid chromatography-tandem mass spectrometry for determination of macrolides in fish tissues. Food Analytical Methods, $6(5), 1448-1457$.

Zhanel, G. G., Dueck, M., Hoban, D. J., Vercaigne, L. M., Embil, J. M., Gin, A. S., \& Karlowsky, J. A. (2001). Review of macrolides and ketolides. Drugs, 61(4), 443-498.

Zhang, J. H., Jiang, M., Zou, L., Shi, D., Mei, S. R., Zhu, Y. X., Shi, Y., Dai, K., \& Lu, B. (2006). Selective solid-phase extraction of bisphenol A using molecularly imprinted polymers and its application to biological and environmental samples. Anal Bioanal Chem, 385(4), 780-786.

Zhang, Z., Yang, X., Zhang, H., Zhang, M., Luo, L., Hu, Y., \& Yao, S. (2011). Novel molecularly imprinted polymers based on multi-walled carbon nanotubes with binary functional monomer for the solid-phase extraction of erythromycin from chicken muscle. Journal of Chromatography B, 879(19), 1617-1624.

Zhao, X.T., Lin, Q.B., Song, H., Pan, Y.L., \& Wang, X. (2011). Development of an immunoaffinity chromatography purification and ultra performance liquid chromatography tandem mass spectrometry method for determination of 12 sulfonamides in beef and milk. Journal of agricultural and food chemistry, 59(18), 9800-9805.

Zheng, Y., Liu, Y., Guo, H., He, L., Fang, B., \& Zeng, Z. (2011). Molecularly imprinted solid-phase extraction for determination of tilmicosin in feed using high performance liquid chromatography. Analytica chimica acta, 690(2), 269-274.

Zhou, J., Chen, Y., \& Cassidy, R. (2000). Separation and determination of the macrolide antibiotics (erythromycin, spiramycin and oleandomycin) by capillary electrophoresis coupled with fast 
reductive voltammetric detection. Electrophoresis, 21(7), 1349-1353.

533 
Figure 1 Influence of methanol, ethyl acetate and different proportion of acetonitrile in water as loading solvent on the recoveries of macrolides.

Figure 2 Influence of methanol, acetonitrile and ethyl acetate on extraction

541 efficiency of ten macrolides at the spiked concentration of $5 \mu \mathrm{g} \mathrm{kg}^{-1}$ in swine muscle.

Figure 3 Influence of the different types of SPE cartridges on the recoveries of ten macrolides at the spiked concentration of $5 \mu \mathrm{gg}^{-1}$ in swine muscle matrix.

Figure 4 Typical MRM chromatograms from the extracts of blank swine matrix at the spiked concentration of $5 \mu \mathrm{gg}^{-1}$ (A) and blank swine matrix (B).

Figure S1 The chemical structures of ten macrolide drugs.

Figure S2 The MRM chromatograms from the extracts of blank cattle (A) and blank chicken (B) muscles matrices.

Figure S3 The MRM chromatograms with electronic ionspray source in positive 

concentration of the LOQ of each macrolide drugs.

558 Figure S4 The MRM chromatograms with electronic ionspray source in positive mode achieved from the extracts of blank cattle and chicken muscles matrices at the spiked concentration of the LOQ of each macrolide drugs. 


\section{Tables}

\section{Table 1}

The optimum parameters of LC-MS/MS for ten macrolides in electronic spray positive ion mode

\begin{tabular}{|c|c|c|c|c|c|c|c|c|}
\hline \multirow[t]{2}{*}{ Compound } & \multirow[t]{2}{*}{ Abbr. $^{a}$} & \multirow{2}{*}{$\begin{array}{l}\text { Precursor } \\
\text { ion }(\mathrm{m} / \mathrm{z})\end{array}$} & \multirow{2}{*}{$\begin{array}{l}\text { Product } \\
\text { ion }(\mathrm{m} / \mathrm{z})\end{array}$} & \multirow[t]{2}{*}{$\mathrm{DP}(\mathrm{V})^{c}$} & \multirow[t]{2}{*}{$\mathrm{CE}(\mathrm{eV})^{d}$} & \multicolumn{2}{|c|}{ Ion ratio $(\mathrm{RSD}, \%)^{e}$} & \multirow{2}{*}{$\begin{array}{c}\text { relative } \\
\text { Difference (\% }\end{array}$} \\
\hline & & & & & & pure solvent & muscle matrix & \\
\hline \multirow[t]{2}{*}{ Azithromycin } & AZI & 749.7 & $158.2^{b}$ & 80 & 46 & $46.6(1.1)$ & $44.6(1.7)$ & 4.4 \\
\hline & & & 591.8 & 75 & 28 & & & \\
\hline \multirow[t]{2}{*}{ Tulathromycin } & TUL & 404.0 & $72.2^{b}$ & 71 & 31 & $87.3(0.3)$ & $88.6(0.4)$ & 1.5 \\
\hline & & & 158.2 & 71 & 33 & & & \\
\hline \multirow[t]{2}{*}{ Spiramycin } & SPM & 843.9 & $174.4^{b}$ & 110 & 50 & $26.6(0.7)$ & $25.6(0.8)$ & 3.7 \\
\hline & & & 141.9 & 110 & 48 & & & \\
\hline \multirow[t]{2}{*}{ Tilmicosin } & TIL & 869.6 & $174.2^{b}$ & 130 & 60 & $46.4(0.6)$ & $49.6(0.4)$ & 6.9 \\
\hline & & & 696.4 & 130 & 66 & & & \\
\hline
\end{tabular}




\begin{tabular}{|c|c|c|c|c|c|c|c|c|}
\hline \multirow[t]{2}{*}{ Erythromycin } & ERY & 734.7 & $158.0^{b}$ & 64 & 43 & $69.6(1.1)$ & $64.2(0.4)$ & 7.8 \\
\hline & & & 576.5 & 64 & 27 & & & \\
\hline \multirow[t]{2}{*}{ Clarithromycin } & CLA & 749.6 & $158.1^{b}$ & 80 & 41 & $61.7(1.1)$ & $56.3(0.5)$ & 8.7 \\
\hline & & & 591.5 & 80 & 26 & & & \\
\hline \multirow[t]{2}{*}{ Roxithromycin } & ROX & 837.8 & $679.7^{b}$ & 85 & 31 & $23.9(2.1)$ & $24.5(1.7)$ & 2.6 \\
\hline & & & 158.1 & 85 & 48 & & & \\
\hline \multirow[t]{2}{*}{ Medecamycin } & MED & 814.8 & $174.3^{b}$ & 90 & 46 & $15.7(2.0)$ & $16.1(2.3)$ & 2.2 \\
\hline & & & 108.6 & 90 & 34 & & & \\
\hline \multirow[t]{2}{*}{ Josamycin } & JOS & 829.4 & $174.3^{b}$ & 100 & 48 & $26.6(3.4)$ & $25.9(0.9)$ & 2.4 \\
\hline & & & 229.5 & 100 & 44 & & & \\
\hline \multirow[t]{2}{*}{ Kitasamycin } & KIT & 772.8 & $174.1^{b}$ & 100 & 45 & $75.4(0.5)$ & $72.2(0.4)$ & 4.3 \\
\hline & & & 215.1 & 100 & 41 & & & \\
\hline
\end{tabular}

${ }^{a}$ Abbr., abbreviations. ${ }^{b}$ Product ion for quantification. ${ }^{c} \mathrm{DP}$, declustering potential. ${ }^{d} \mathrm{CE}$, collision energy. ${ }^{e}$ Ion ratio(\%), the ion ratio in pure solvent and swine muscle matrix at the spiked concentration of $5 \mu \mathrm{g} \mathrm{L}{ }^{-1}$. 
Table 2

Linearity, LOD, LOQ, recovery and precision of ten macrolides in different muscles samples at three spiked concentration levels ${ }^{a}$

\begin{tabular}{|c|c|c|c|c|c|c|c|c|c|c|c|}
\hline \multirow[b]{2}{*}{ Compound } & \multirow[b]{2}{*}{ Abbr. } & \multirow[b]{2}{*}{ Muscle } & \multirow[b]{2}{*}{ Linearity (r) } & \multirow[b]{2}{*}{$\mathrm{LOD}\left(\mu \mathrm{g} \mathrm{kg}{ }^{-1}\right)$} & \multirow[b]{2}{*}{$\mathrm{LOQ}\left(\mu \mathrm{g} \mathrm{kg}{ }^{-1}\right)$} & \multicolumn{3}{|c|}{ Intra-day recovery (\%RSD, n=6) } & \multicolumn{3}{|c|}{ Inter-day recovery (\%RSD, $\mathrm{n}=18$ ) } \\
\hline & & & & & & $1 \mu \mathrm{g} \mathrm{kg}{ }^{-1}$ & $5 \mu \mathrm{gg}^{-1}$ & $20 \mu \mathrm{g} \mathrm{kg}^{-1}$ & $1 \mu \mathrm{g} \mathrm{kg}{ }^{-1}$ & $5 \mu \mathrm{kg}^{-1}$ & $20 \mu \mathrm{g} \mathrm{kg}^{-1}$ \\
\hline \multirow[t]{3}{*}{ Azithromycin } & AZI & swine & 0.9973 & 0.3 & 1.0 & $73.0(2.4)$ & $75.8(3.7)$ & $75.6(4.1)$ & $72.4(2.4)$ & $75.6(4.2)$ & $74.8(4.6)$ \\
\hline & & cattle & 0.9925 & 0.3 & 1.0 & $76.3(4.3)$ & $70.4(8.3)$ & $89.7(8.6)$ & 76.0 (3.9) & $73.7(8.5)$ & $89.2(9.8)$ \\
\hline & & chicken & 0.9934 & 0.3 & 1.0 & $72.9(10)$ & $71.7(8.8)$ & $96.0(6.8)$ & $68.7(12)$ & $73.2(12)$ & $89.7(13)$ \\
\hline \multirow[t]{3}{*}{ Tulathromycin } & TUL & swine & 0.9988 & 0.3 & 1.0 & $65.4(3.5)$ & $66.3(6.0)$ & $73.2(2.8)$ & $67.9(3.5)$ & $68.7(6.8)$ & $71.8(1.7)$ \\
\hline & & cattle & 0.9978 & 0.3 & 1.0 & $60.9(0.5)$ & $69.9(3.4)$ & $61.5(2.4)$ & $63.3(5.0)$ & $66.4(6.0)$ & $70.0(6.4)$ \\
\hline & & chicken & 0.9995 & 0.3 & 1.0 & 60.7 (9.3) & 68.3 (3.9) & $62.0(11)$ & $63.6(10)$ & $64.2(1.7)$ & $68.0(7.6)$ \\
\hline \multirow[t]{3}{*}{ Spiramycin } & SPM & swine & 0.9995 & 0.1 & 0.3 & $64.9(12)$ & $67.1(6.2)$ & $69.0(2.2)$ & $62.9(12)$ & 79.5 (8.2) & $66.1(6.4)$ \\
\hline & & cattle & 0.9978 & 0.1 & 0.3 & $61.0(5.0)$ & 62.9 (4.9) & $71.8(4.0)$ & $63.9(6.4)$ & $64.2(4.9)$ & $68.2(7.0)$ \\
\hline & & chicken & 0.9997 & 0.1 & 0.3 & 79.9 (2.2) & $63.3(1.5)$ & 75.4 (5.9) & 78.5 (2.4) & $64.0(3.5)$ & 73.8 (5.1) \\
\hline
\end{tabular}




\begin{tabular}{|c|c|c|c|c|c|c|c|c|c|c|c|}
\hline \multirow[t]{3}{*}{ Tilmicosin } & TIL & swine & 0.9995 & 0.2 & 0.6 & $70.5(2.0)$ & $75.3(4.1)$ & $68.4(8.2)$ & $64.1(4.6)$ & 74.9 (5.9) & 79.7 (10) \\
\hline & & cattle & 0.9986 & 0.2 & 0.6 & $70.2(9.0)$ & $69.0(4.5)$ & $68.3(2.6)$ & $76.3(5.0)$ & $65.0(5.6)$ & $70.2(3.6)$ \\
\hline & & chicken & 0.9978 & 0.2 & 0.6 & $67.6(2.7)$ & $63.0(3.4)$ & $85.0(14)$ & $68.0(3.4)$ & $66.6(10)$ & $85.9(14)$ \\
\hline \multirow[t]{3}{*}{ Erythromycin } & ERY & swine & 0.9981 & 0.4 & 1.0 & $88.6(1.7)$ & 85.7 (5.6) & $85.2(5.2)$ & $85.9(4.6)$ & 84.4 (5.9) & $78.4(10)$ \\
\hline & & cattle & 0.9998 & 0.4 & 1.0 & $75.7(0.2)$ & $93.6(4.6)$ & $90.6(7.1)$ & $74.2(5.0)$ & $94.4(4.2)$ & $97.0(8.4)$ \\
\hline & & chicken & 0.9998 & 0.4 & 1.0 & $87.2(3.2)$ & $87.7(4.6)$ & $86.8(5.6)$ & 94.3 (8.9) & $92.9(8.4)$ & $86.9(7.6)$ \\
\hline \multirow[t]{3}{*}{ Clarithromycin } & CLA & swine & 0.9985 & 0.1 & 0.3 & 64.8 (5.3) & $82.3(4.9)$ & 82.7 (6.7) & $68.8(7.4)$ & $82.1(5.0)$ & 82.7 (7.3) \\
\hline & & cattle & 0.9957 & 0.2 & 0.5 & 73.0 (3.8) & 74.9 (2.6) & $85.0(5.7)$ & 80.1 (8.9) & 77.7 (10) & $85.0(6.5)$ \\
\hline & & chicken & 0.9916 & 0.2 & 0.5 & 75.7 (9.4) & $71.0(1.4)$ & $88.1(3.0)$ & $72.4(10)$ & $67.2(5.4)$ & $88.4(7.5)$ \\
\hline \multirow[t]{3}{*}{ Medecamycin } & MED & swine & 0.9982 & 0.1 & 0.3 & $93.9(4.2)$ & $89.4(5.8)$ & $92.0(2.4)$ & $96.5(4.5)$ & $89.1(6.1)$ & $89.0(5.5)$ \\
\hline & & cattle & 0.9996 & 0.1 & 0.3 & 79.7 (6.3) & $85.6(5.3)$ & 85.3 (3.5) & $80.2(7.3)$ & $84.2(9.5)$ & 86.6 (3.3) \\
\hline & & chicken & 0.9987 & 0.1 & 0.3 & $81.2(4.1)$ & $92.2(10)$ & $100.3(4.0)$ & $85.9(12)$ & 84.5 (11) & $96.8(5.8)$ \\
\hline \multirow[t]{2}{*}{ Josamycin } & JOS & swine & 0.9961 & 0.2 & 0.5 & $87.3(1.4)$ & 83.5 (3.8) & 87.5 (3.3) & 89.0 (4.9) & $85.9(4.4)$ & 85.8 (3.6) \\
\hline & & cattle & 0.9945 & 0.2 & 0.5 & $79.2(2.6)$ & $72.6(2.0)$ & 77.4 (7.6) & 74.8 (5.7) & $74.3(5.5)$ & $84.2(10)$ \\
\hline
\end{tabular}




\begin{tabular}{|c|c|c|c|c|c|c|c|c|c|c|c|}
\hline & & chicken & 0.9934 & 0.2 & 0.5 & 76.7 (2.7) & $91.5(11)$ & $90.0(2.5)$ & $88.7(12)$ & $81.2(7.1)$ & 84.9 (9.1) \\
\hline \multirow[t]{3}{*}{ Kitasamycin } & KIT & swine & 0.9992 & 0.2 & 0.5 & $88.6(1.4)$ & $82.6(4.2)$ & $82.8(1.8)$ & $91.3(4.0)$ & $82.0(4.2)$ & $84.4(3.2)$ \\
\hline & & cattle & 0.9957 & 0.2 & 0.5 & $76.7(5.5)$ & $82.3(5.0)$ & $82.2(1.7)$ & $75.7(6.0)$ & $85.3(7.4)$ & $81.0(5.4)$ \\
\hline & & chicken & 0.9996 & 0.2 & 0.5 & 84.8 (8.6) & $83.0(2.1)$ & $88.3(7.5)$ & $88.7(10)$ & $83.4(4.4)$ & $92.1(13)$ \\
\hline \multirow[t]{3}{*}{ Roxithromycin } & ROX & swine & 0.9969 & 0.4 & 1.0 & 72.5 (3.6) & $71.1(1.7)$ & 83.1 (3.8) & 73.1 (3.8) & 73.6 (4.8) & 79.7 (5.7) \\
\hline & & cattle & 0.9978 & 0.4 & 1.0 & 75.6 (3.6) & $73.5(1.2)$ & $81.7(2.6)$ & $78.6(4.6)$ & 70.6 (5.7) & $81.5(4.9)$ \\
\hline & & chicken & 0.9927 & 0.4 & 1.0 & $73.0(8.1)$ & $71.9(0.6)$ & 91.3 (5.6) & 72.5 (9.9) & $72.1(5.2)$ & $90.2(11)$ \\
\hline
\end{tabular}

${ }^{a}$ Abbr., abbreviations; LOD, limit of detection; LOQ, limit of quantification; RSD, relative standard deviation. 


\section{FIGURES}

\section{Figure 1}

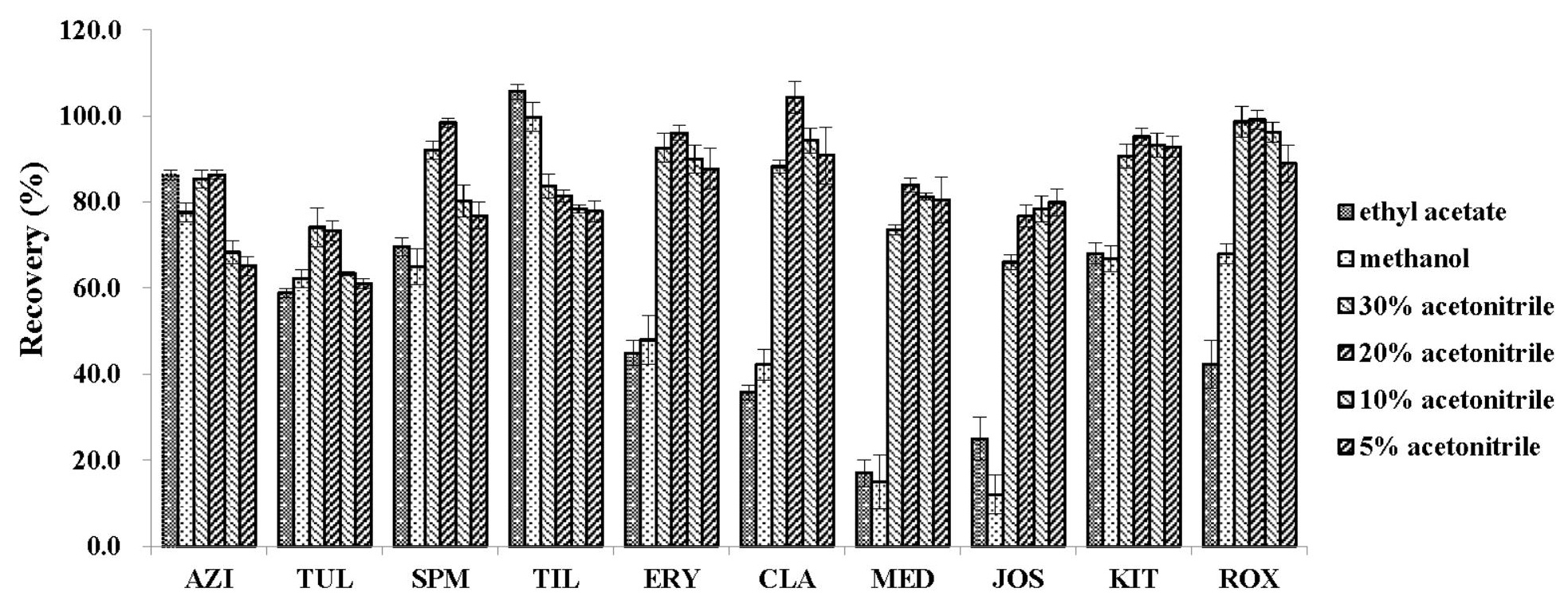




\section{Figure 2}

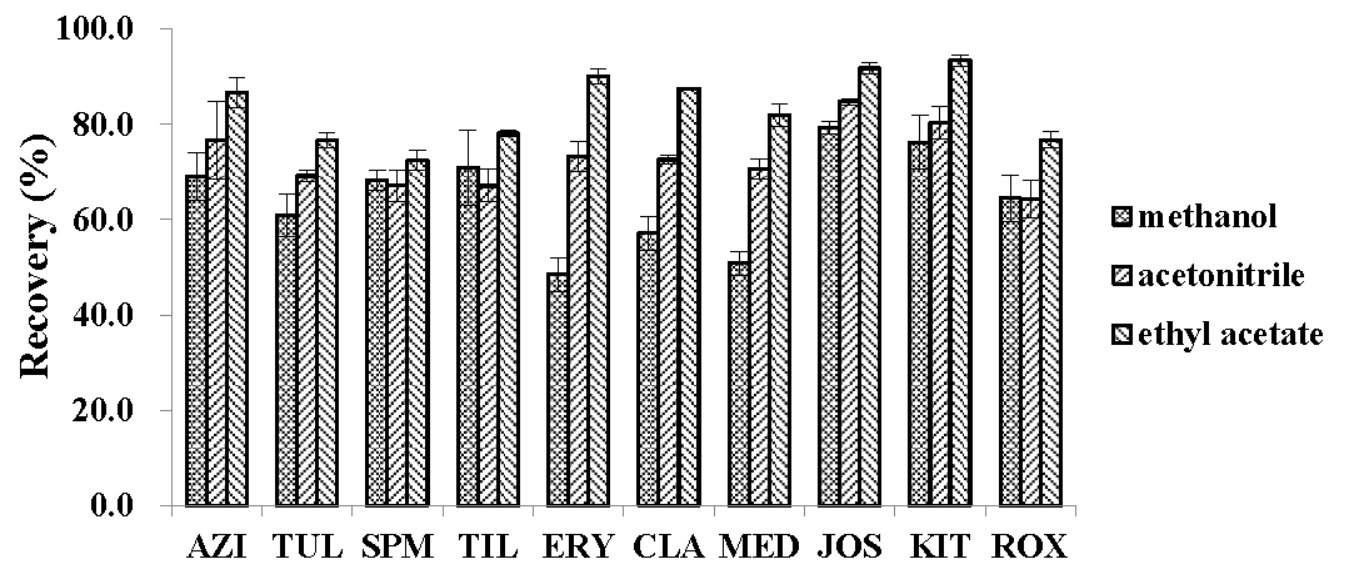


Figure 3

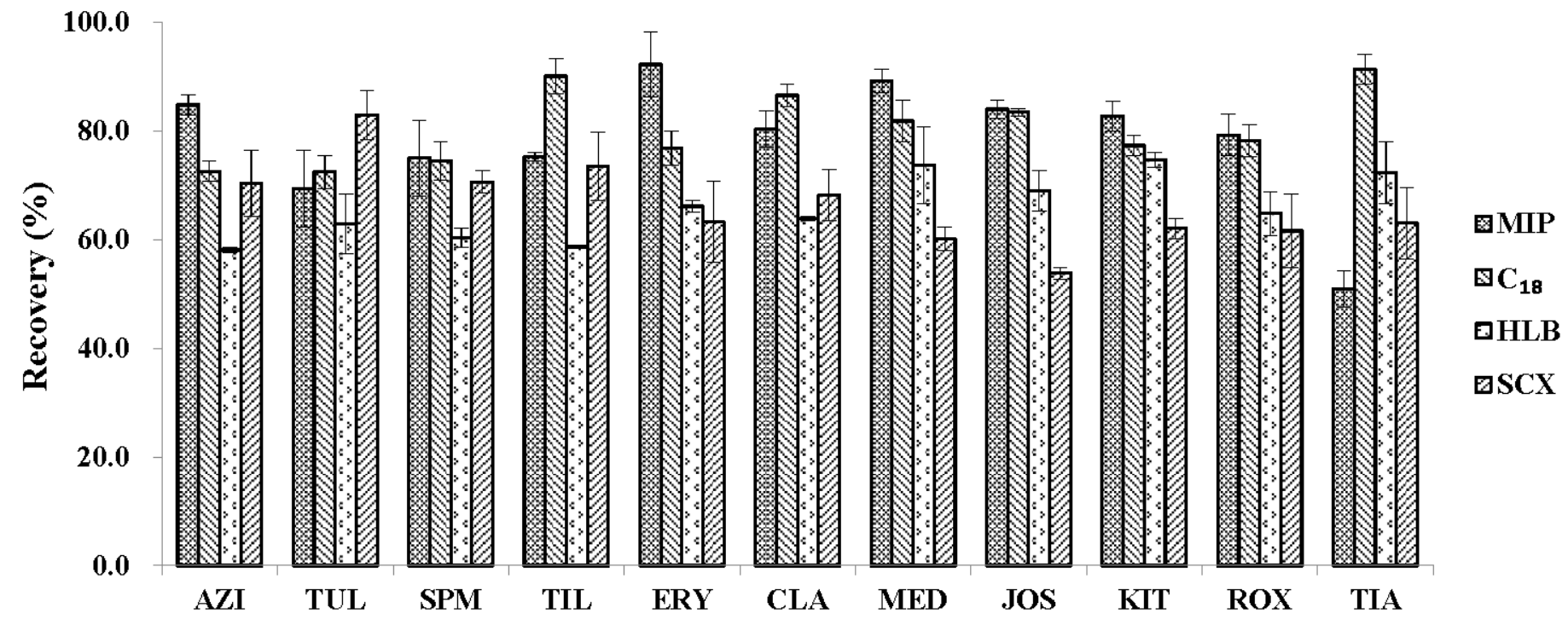


Figure 4 $\mathbf{A}$

AZI 749.7/158.2

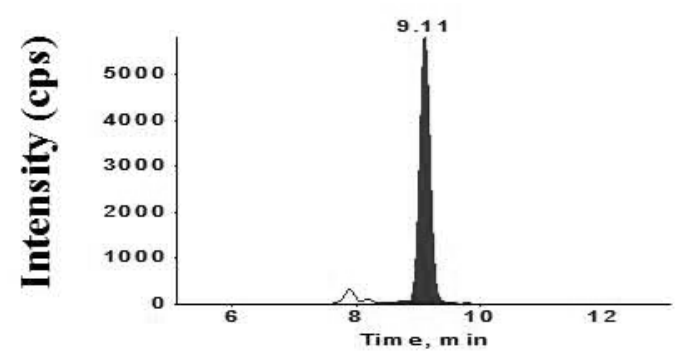

ERY 734.7/158.0

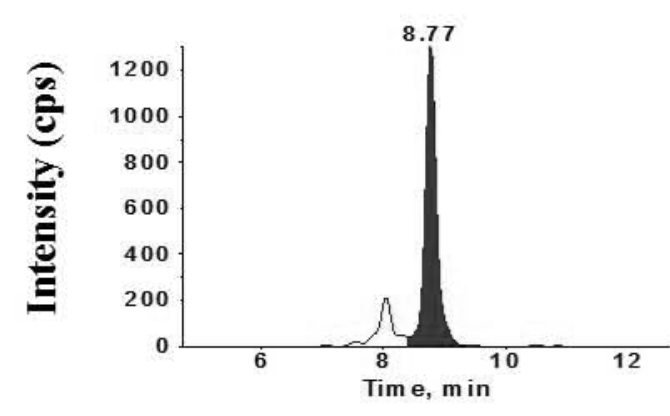

B

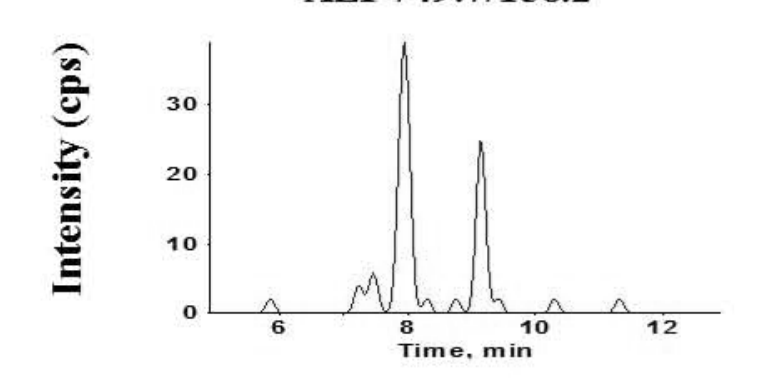

ERY 734.7/158.0

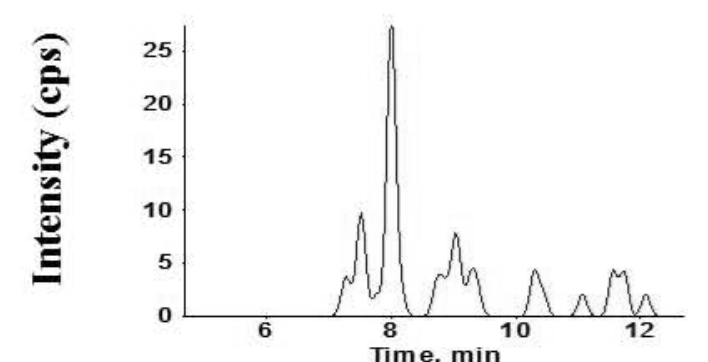

TUL 404/72.2

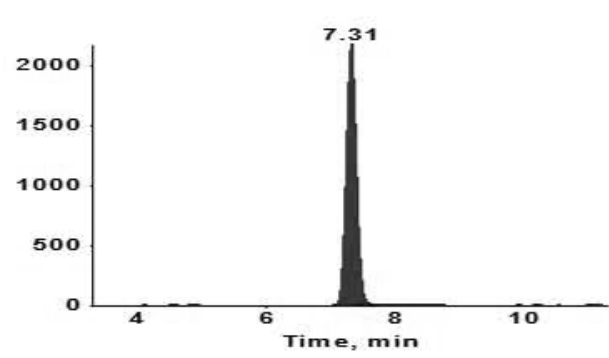

CLA 749.6/158.1

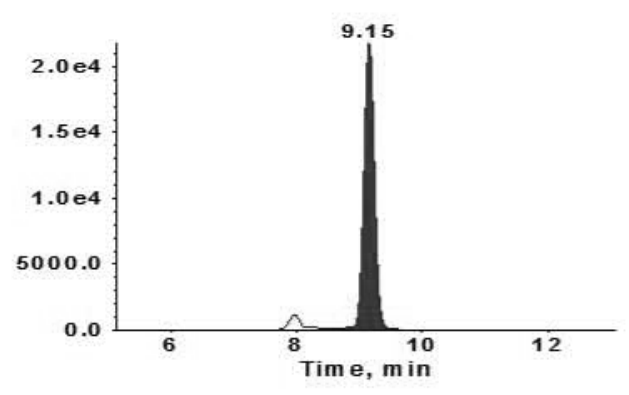

TUL 404/72.2

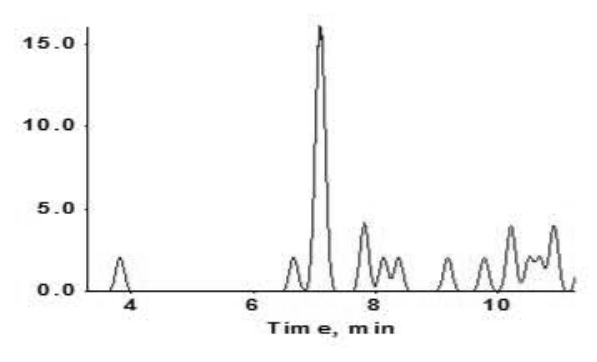

CLA 749.6/158.1

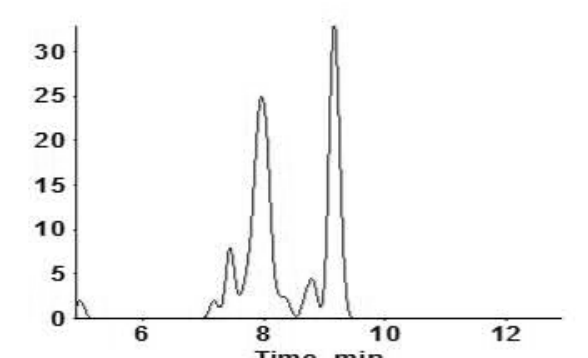

SPM 843.9/174.4

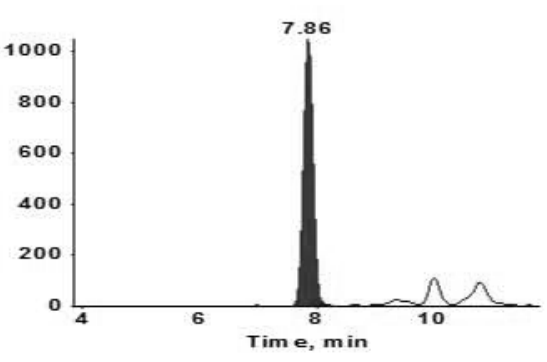

MED 814.8/174.3

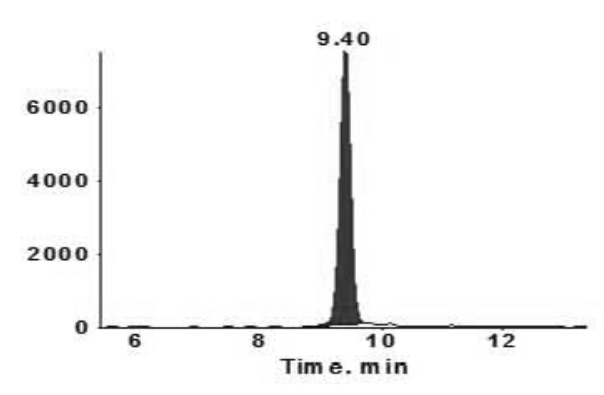

SPM 843.9/174.4

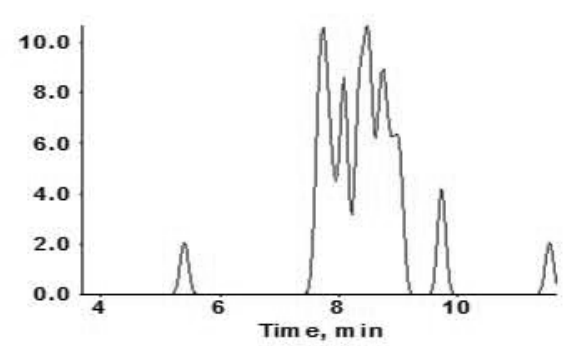

MED 814.8/174.3

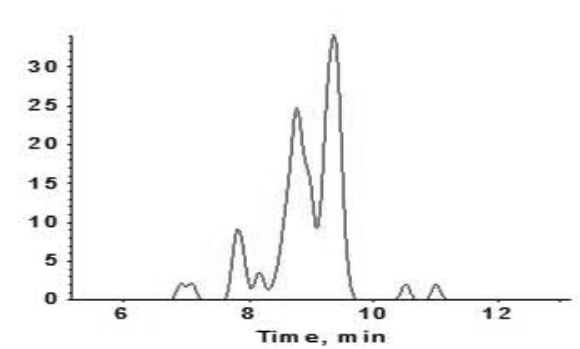

TIL 869.6/174.2

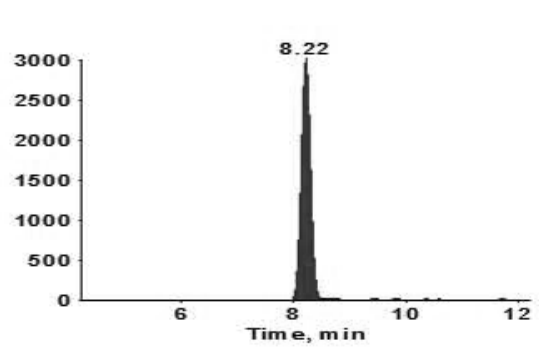

JOS 829.4/174.3

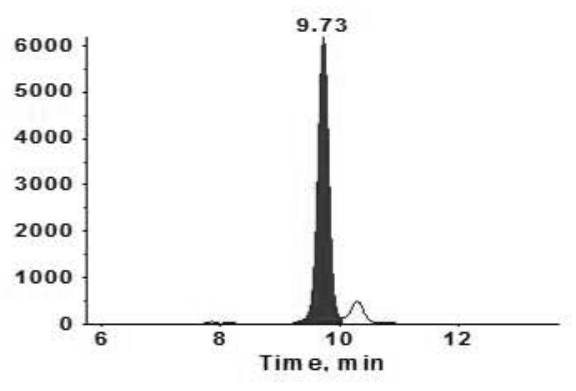

TIL 869.6/174.2

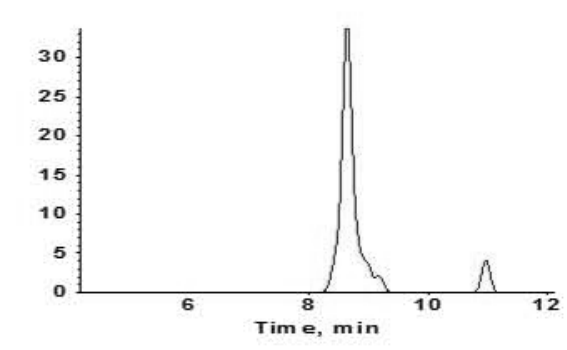

JOS 829.4/174.3

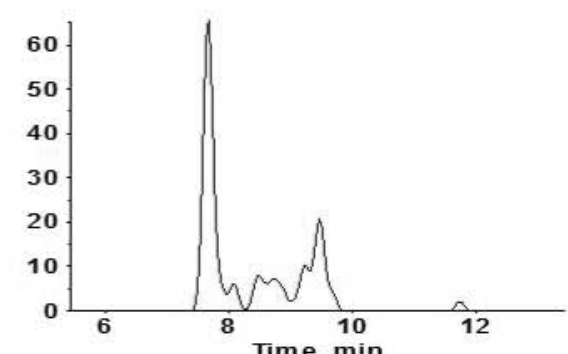

ROX 837.8/679.7

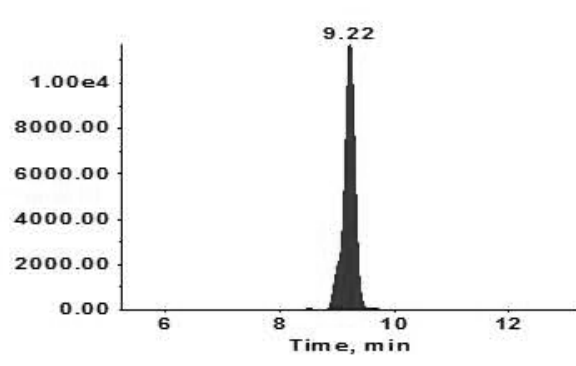

KIT 772.8/174.1

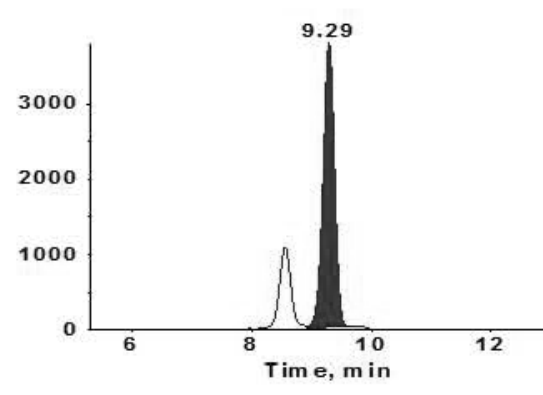

ROX 837.8/679.7

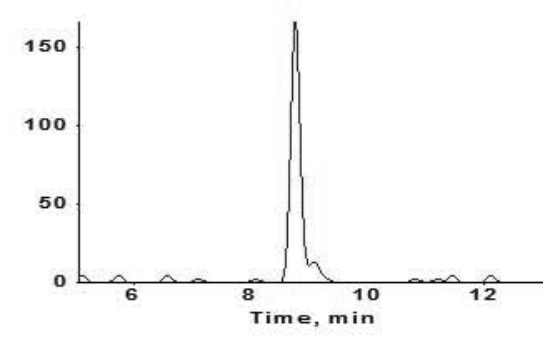

KIT 772.8/174.1

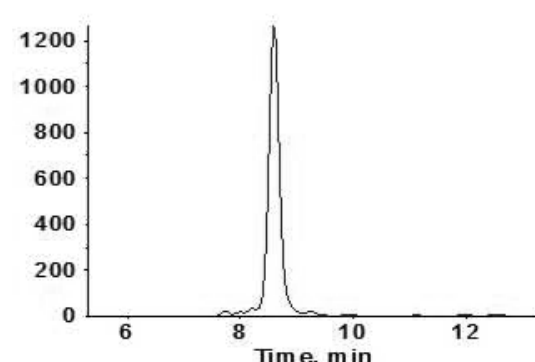

